\title{
BMJ Open Using a simple point-prevalence survey to define appropriate antibiotic prescribing in hospitalised children across the UK
}

\author{
Myriam Gharbi, ${ }^{1}$ Katja Doerholt, ${ }^{2}$ Stefania Vergnano, ${ }^{3}$ Julia Anna Bielicki, ${ }^{3}$ \\ Stéphane Paulus, ${ }^{4}$ Esse Menson, ${ }^{5}$ Andrew Riordan, ${ }^{4}$ Hermione Lyall, ${ }^{6}$ \\ Sanjay Valabh Patel, ${ }^{7}$ Jolanta Bernatoniene, ${ }^{8}$ Ann Versporten, ${ }^{9}$ \\ Maggie Heginbothom, ${ }^{10}$ Herman Goossens, ${ }^{9}$ Mike Sharland, ${ }^{2,3}$ for the ARPEC \\ project Group members
}

To cite: Gharbi M, Doerholt $\mathrm{K}$, Vergnano S, et al. Using a simple pointprevalence survey to define appropriate antibiotic prescribing in hospitalised children across the UK. BMJ Open 2016;6:e012675. doi:10.1136/bmjopen-2016012675

- Prepublication history and additional material is available. To view please visit the journal (http://dx.doi.org/ 10.1136/bmjopen-2016012675).

Received 17 May 2016 Revised 27 July 2016 Accepted 5 September 2016

CrossMark

For numbered affiliations see end of article.

Correspondence to Dr Myriam Gharbi; m.gharbi@yahoo.fr

\section{ABSTRACT}

Background: The National Health Service England, Commissioning for Quality and Innovation for Antimicrobial Resistance (CQUIN AMR) aims to reduce the total antibiotic consumption and the use of certain broad-spectrum antibiotics in secondary care.

However, robust baseline antibiotic use data are lacking for hospitalised children. In this study, we aim to describe, compare and explain the prescription patterns of antibiotics within and between paediatric units in the UK and to provide a baseline for antibiotic prescribing for future improvement using CQUIN AMR guidance.

Methods: We conducted a cross-sectional study using a point prevalence survey (PPS) in 61 paediatric units across the UK. The standardised study protocol from the Antibiotic Resistance and Prescribing in European Children (ARPEC) project was used. All inpatients under 18 years of age present in the participating hospital on the day of the study were included except neonates.

Results: A total of $1247(40.9 \%)$ of 3047 children hospitalised on the day of the PPS were on antibiotics. The proportion of children receiving antibiotics showed a wide variation between both district general and tertiary hospitals, with $36.4 \%$ ( $95 \% \mathrm{Cl} 33.4 \%$ to $39.4 \%)$ and $43.0 \%(95 \% \mathrm{Cl} 40.9 \%$ to $45.1 \%)$ of children prescribed antibiotics, respectively. About a quarter of children on antibiotic therapy received either a medical or surgical prophylaxis with parenteral administration being the main prescribed route for antibiotics (>60\% of the prescriptions for both types of hospitals). General paediatrics units were surprisingly high prescribers of critical broad-spectrum antibiotics, that is, carbapenems and piperacillin-tazobactam.

Conclusions: We provide a robust baseline for antibiotic prescribing in hospitalised children in relation to current national stewardship efforts in the UK. Repeated PPS with further linkage to resistance data needs to be part of the antibiotic stewardship strategy to tackle the issue of suboptimal antibiotic use in hospitalised children.

\section{Strengths and limitations of this study}

We used a simple, rigorous, validated and standardised point prevalence method to provide the baseline for antimicrobial prescribing in hospitalised children to assess current and future national strategies in the UK.

- Data were collected from a large sample of hospitalised children on antibiotics $(n=1247)$ including a wide variety of different hospitals (61 institutions) across the UK, wards and patient characteristics.

- Data were collected at the patient level providing information on the paediatric antimicrobial prescribing in secondary care adjusted on the case mix.

- Only volunteer hospitals were including in this cross-sectional study leading to potential selection biases and limited temporal relationship between antimicrobial prescribing and covariates.

- No consensus exists for measuring antibiotic prescribing in children as defined daily doses/ 100 inpatients is not a validated measure for this population.

\section{INTRODUCTION}

The increasing levels of antimicrobial resistance (AMR) are strongly correlated with inappropriate use of antibiotics. ${ }^{1}{ }^{2}$ Recent UK and international reports have advocated the critical need to monitor and control the use of existing antibiotics since the number of new classes of antibiotics has dramatically decreased over the past 40 years. ${ }^{3-5}$ Antimicrobial stewardship programmes (ASPs), defined as comprehensive quality improvement activities for optimising antimicrobial prescribing and minimising resistance, have been widely adopted in adult care 
settings, ${ }^{6}{ }^{7}$ but still remain limited in children's units. ${ }^{8} 9$ The heterogeneity in age and weight of children, as well as the lack of a standardised method to quantify antibiotic use in paediatrics, increases the challenge of determining and benchmarking the appropriateness of prescribing within or between children institutions, ${ }^{10-12}$ and children are often excluded from comparative studies on antibiotic use. ${ }^{13} 14$

The National Health Service England, Commissioning for Quality and Innovation for Antimicrobial Resistance (AMR CQUIN) 2016/2017, aims to reduce by $1 \%$ or more per year the total antibiotic consumption and the use of certain broad-spectrum antibiotics considered as critical antibiotics (carbapenems and piperacillintazobactam) in secondary care. ${ }^{15-17}$ However, robust baseline antibiotic use data, so far developed for adults, are lacking for hospitalised children while they are key to measure the impact of the proposed strategies and to identify room for improvement. Two international studies have proposed to describe and compare the use of antimicrobials in children across Europe and worldwide using various quality indicators, ${ }^{18}{ }^{19}$ but no comparable detailed information on antibiotic use in hospitalised children in the UK is available.

The aim of our study is to describe, compare and explain the prescription pattern of antibiotics across paediatric units in the UK collected in a cross-sectional point prevalence survey (PPS) carried out as part of the Antibiotic Resistance and Prescribing in European Children (ARPEC) project. ${ }^{20}{ }^{21}$ We also proposed to use the simple PPS to apply AMR CQUIN quality indicators to provide a baseline of antibiotic prescribing in children to measure the impact of the current and future national strategies.

\section{METHODS}

\section{Study design and settings}

Detailed antimicrobial prescribing data were collected for all inpatients aged under 18 years present in a participating hospital's paediatric and neonatal wards at 08:00 since at least midnight. Data collection included a wide variety of different hospitals, wards and patient characteristics to be as representative as possible of hospitalised children in the UK. Data were collected on paper forms, anonymously entered, validated and reported online through the ARPEC-PPS programme. Information on surgical prophylaxis was captured for the previous 24 hours. Antimicrobial agents were analysed in accordance with the Anatomical Therapeutic Chemical (ATC) Classification. ${ }^{22}$ To facilitate the data collection on underlying diagnosis (defined as a pre-existing comorbidity in addition to the diagnosis of infection for which patients are prescribed antibiotics) and reason for treatment with antibiotics, predefined lists of grouped items were used. ${ }^{23}$ The full method is described elsewhere by Versporten $e t a l^{21}$

\section{Data extraction}

For this study, we extracted and analysed data from 61 paediatric units in the UK which participated in the ARPEC-PPS organised in March to April 2011 (feasibility survey), September to November 2011 (worldwide pilot ARPEC-PPS) $^{21}$ and October to December 2012 (full worldwide ARPEC-PPS). ${ }^{19}$ All inpatients under 18 years of age admitted to a paediatric ward were included. We excluded infants on neonatal units and those on children's wards under 28 days of age. We analysed antibacterials for systemic use (ATC J01).

\section{Data analysis}

\section{Descriptive analysis}

Demographic data, presence or not of an underlying chronic condition, current diagnosis, hospital-acquired infections versus community-acquired infections (CAIs), therapeutic versus prophylactic prescribing, and antibiotic type, dosing and route of administration were analysed and compared between 44 district general hospitals, which provide secondary care, and 17 tertiary referral hospitals, which provide tertiary or specialised care.

\section{Metrics for measuring antibiotic use}

We compared two different metrics of antibiotic prescribing within and between hospitals: (1) the proportion of children on antibiotics (prevalence rate) with $95 \%$ CIs ; (2) the defined daily doses per 100 inpatients (DDD/100 inpatients), as recommended in the AMR CQUIN. ${ }^{17} 24$ Antibiotic consumption in grams was converted into DDD using the 2013 release of the ATC Classification. $^{22}$ The denominator 'inpatients' was defined in this study as the sum of inpatients in the hospital at 08:00.

\section{Quality indicators for national benchmarking between UK hospitals}

We explored the different inpatient antibiotic prescribing quality indicators proposed by CQUIN NHS England for AMR. ${ }^{17}$

1. The total amount of antibiotics prescribed using both metrics, the proportion of children receiving antibiotics and DDD/100 inpatients in different age bands. A funnel plot was used to graphically compare antibiotic prescribing between hospitals, to adjust for different hospital sizes and to identify outliers. ${ }^{25}$ This takes account of the variable number of cases by institution by plotting the proportion of children on antibiotics against the sample size for each hospital using a binomial distribution and 95\% CI $(\sim 2 \mathrm{SD})$. We also displayed antibiotic prescribing in DDD/100 inpatients for each hospital, as well as the median and IQR for each age band.

2. The use of the carbapenems and the use of piperacillin-tazobactam, which are both considered critically important antibiotics against extendedspectrum $\beta$-lactamase producing Gram-negative 
bacteria. ${ }^{3}$ The proportions of children on carbapenems and piperacillin-tazobactam, as well as the amount of these drugs prescribed in DDD/100 inpatients, were monitored and compared between institutions after adjusting for hospital type (district general hospitals vs tertiary referral hospitals) and presence of underlying disease.

\section{Statistical analyses}

We conducted comparative analyses to determine the balance between district general hospitals and tertiary referral hospitals using tests of proportions (eg, $\chi^{2}$ analysis, Fisher's exact test) and tests of central tendency (eg, analysis of variance, sign rank). Mean total daily doses were compared by an unpaired two-sample t-test. All $\mathrm{p}$ values were based on a two-tailed test with $\mathrm{p}$ value $<0.05$ for significance. Statistical analysis was performed using STATA V.12 (STATA Corp, College Station, Texas, USA).

\section{Ethics}

The responsible UK Research Ethics Committee was approached to establish the need for a formal evaluation. Written confirmation was provided that within the UK framework a fully anonymised PPS constituted surveillance and that formal review by the Research Ethics Committee was not required.

\section{RESULTS}

\section{Patient demographics}

A total of 1247 (40.9\%) of 3047 surveyed UK paediatric inpatients were receiving antimicrobials. Overall, 1348 indications were recorded for 1247 inpatients with a total of 1858 antibiotic prescriptions. The median age of exposed children was 2 years $(\mathrm{IQR}=0.083-8)$. More than two-thirds of inpatients were recruited from tertiary care centres, and from general paediatric and paediatric surgery wards (see online supplementary table).

Age differences by specialty were seen among children on antibiotics. For general paediatrics, the median age of exposed children was 2 years $(\mathrm{IQR}=0.75-6)$, for surgery 5 years $(\mathrm{IQR}=1.25-11)$, for paediatric intensive care units (PICU) 0.71 years $(\mathrm{IQR}=0.08-3)$, for haematology-oncology-transplant 6 years $(\mathrm{IQR}=2-11)$ and for other medical specialties 3 years $(\mathrm{IQR}=0.75-9)$.

\section{Total use of antibiotics}

\section{Proportion of children on antibiotics}

Table 1 shows that the proportion of children on antibiotics and the number of prescribed antibiotics was significantly higher in tertiary hospitals $(43.0 \%, 95 \%$ CI $40.9 \%$ to $45.1 \%$, 40 different prescribed antibiotics) than in district general hospitals $(36.4 \%, 95 \%$ CI $33.4 \%$ to $39.4 \%, 30$ different prescribed antibiotics, $\mathrm{p}=0.001$ ). About two-thirds of inpatients in intensive or specialist care wards (PICU and haematology-oncology-transplant) were prescribed antibiotics in high specialist care areas compared with about one-third in general paediatrics and surgery. Multiple antibiotics were also used more frequently in children admitted to PICU (77/145, $53.1 \%, 95 \%$ CI $45.0 \%$ to $61.2 \%$ ) and haematologyoncology-transplant units $(63 / 92 ; 68.5 \%, 95 \%$ CI $59.0 \%$ to $78.0 \%$ ) compared with children in paediatric surgery (93/214; $43.5 \%$, $95 \%$ CI $36.8 \%$ to $50.1 \%)$ and general paediatrics (199/554, $35.9 \%, 95 \%$ CI $31.9 \%$ to $39.9 \%)$.

Among all children receiving antibiotics, 60.9\% (95\% CI $57.5 \%$ to $64.4 \%$ ) of children had an underlying disease compared with $39.1 \%$ (95\% CI $34.7 \%$ to $43.4 \%$ ) of previously healthy children. Exposed children were more likely to be younger (69.5\% exposed below 7 years of age compared with $30.5 \%$ at 7 years and older).

Of 1348 indications, a diagnosis of lower respiratory, urinary tract, skin and soft tissue, bone or joint infection, fever and gastrointestinal infection was recorded in $42.2 \%$ (CI $38.1 \%$ to $46.3 \%$ ) compared with $18.2 \%$ (CI $13.4 \%$ to $23.0 \%$ ) with a diagnosis of severe infections, that is, sepsis, catheter-related bloodstream infection (CRBSI), central nervous system (CNS) infection or febrile neutropaenia. For exposed children, treatment for CAIs was almost four times more common $(59.1 \%$, CI $55.7 \%$ to $62.5 \%$ ) than for healthcare-associated infection $(15.7 \%$, CI $10.8 \%$ to $20.6 \%)$. Finally, about a quarter of children on antibiotic therapy received either medical $(17.3 \%)$ or surgical $(6.8 \%)$ prophylaxis.

Figure 1 shows the funnel plot of the proportion of children on antibiotic for each institution. Hospitals with a proportion outside the funnel plot's 2 SD control limits are considered to be potential outliers. Seven of the 61 institutions were identified as potential 'high prescribers', 2 district general hospitals (21 children on antibiotics) and 5 tertiary referral hospitals (322 children on antibiotics). For the two district general hospitals, all children on antibiotics were from general paediatric wards, aged under 7 years for $76.2 \%$ of them (mainly aged between 1 and 6), with $52.4 \%$ of them having an underlying disease and $80.1 \%$ with a common bacterial infection (lower respiratory tract infection, urinary tract infection, skin and soft tissue infection, joint bone tissue infection). For the five tertiary hospitals, a high proportion of children on antibiotics $(30.1 \%)$ were from haematology/oncology/transplant units and PICU, with a total of $71.4 \%$ of children having an underlying disease and $22.7 \%$ of them presenting with a severe infection (sepsis/CRBSI/CNS/febrile neutropaenia) while $21.1 \%$ were on medical prophylaxis. In total, $73.9 \%$ of the children were aged below 7 years $(35.4 \%<1$ and $38.5 \%$ between 1 and 6 ).

\section{Proportion of prescriptions for parenteral versus oral administration}

Parenteral was the main prescribed route for administrating antibiotics, with more than $60 \%$ of the prescriptions in district general hospitals and tertiary referral hospitals. Parenteral antibiotics were highly prescribed in PICU $(81.6 \%$ of the prescriptions), for previous 
Table 1 Proportion of children prescribed antibiotics in paediatric acute care settings across the UK (years 2011, 2012)

\begin{tabular}{|c|c|c|c|c|}
\hline & $\begin{array}{l}\mathrm{N} \text { patients treated with } \\
\text { antibiotic }\end{array}$ & $\begin{array}{l}\text { Proportion of children on antibiotic } \\
\text { per cent }(95 \% \mathrm{Cl})\end{array}$ & $\begin{array}{l}\mathrm{N} \text { antibiotic prescriptions (total of } \\
\text { different prescribed antibiotics) }\end{array}$ & $\begin{array}{l}\text { Parenteral administration } \\
\mathbf{n}(\% \text { of prescriptions) }\end{array}$ \\
\hline $\begin{array}{l}\text { District general hospitals } \\
\text { ( } n=958 \text { patients) }\end{array}$ & 349 & 36.4 (33.4 to 39.4 ) & $479(30)$ & $291(60.8)$ \\
\hline $\begin{array}{l}\text { Tertiary referral hospitals } \\
\text { ( } n=2089 \text { patients) }\end{array}$ & 898 & 43.0 (40.9 to 45.1$)$ & $1379(40)$ & $861(62.4)$ \\
\hline General paediatric $(n=1477)$ & 554 & 37.5 (35.0 to 40.0$)$ & $791(37)$ & $467(59.0)$ \\
\hline PICU $(n=226)$ & 145 & 64.2 (57.9 to 70.5$)$ & $228(27)$ & $186(81.6)$ \\
\hline Paediatric surgery $(n=597)$ & 214 & 35.8 (32.0 to 39.6$)$ & 321 (29) & $223(69.5)$ \\
\hline $\begin{array}{l}\text { Haematology-oncology- } \\
\text { transplant }(n=144)\end{array}$ & 92 & 63.9 (56.1 to 71.7$)$ & $156(24)$ & $77(49.4)$ \\
\hline Others $(n=603)$ & 242 & 40.1 (36.2 to 44.0$)$ & $362(31)$ & $199(55.0)$ \\
\hline \multirow[t]{2}{*}{ Total (n patients $=3047$ ) } & 1247 & 40.9 (39.2 to 42.6$)$ & $1858(41)$ & \\
\hline & $\begin{array}{l}N \text { patients treated with } \\
\text { antibiotic }(\mathrm{N}=1247)\end{array}$ & $\begin{array}{l}\text { Proportion among total children on } \\
\text { antibiotics per cent }(95 \% \mathrm{Cl})\end{array}$ & $\begin{array}{l}\mathrm{N} \text { antibiotic prescriptions (total } \\
\text { of different prescribed antibiotics) }\end{array}$ & $\begin{array}{l}\text { Parenteral administration } \\
\mathrm{n} \text { (\% of prescriptions) }\end{array}$ \\
\hline No underlying disease & 487 & 39.1 (34.7 to 43.4$)$ & $689(30)$ & $483(70.1)$ \\
\hline Underlying disease & 760 & 60.9 (57.5 to 64.4$)$ & $1169(41)$ & 669 (57.2) \\
\hline Aged $<1$ year & 347 & 27.8 (23.1 to 32.6$)$ & $500(29)$ & $337(67.4)$ \\
\hline Aged $1-6$ years & 520 & 41.7 (37.5 to 46.0$)$ & $734(31)$ & $413(56.3)$ \\
\hline Aged $7-11$ years & 174 & $14.0(8.8$ to 19.1$)$ & $259(32)$ & $159(61.4)$ \\
\hline \multirow[t]{2}{*}{ Aged $>12$ years } & 206 & 16.5 (11.4 to 21.5$)$ & $363(36)$ & $243(66.9)$ \\
\hline & $\begin{array}{l}N \text { indications for } \\
\text { antibiotics }(\mathrm{N}=1348)\end{array}$ & Proportion per cent $(95 \% \mathrm{Cl})$ & $\begin{array}{l}\mathrm{N} \text { antibiotic prescriptions (total } \\
\text { of different prescribed antibiotics) }\end{array}$ & $\begin{array}{l}\text { Parenteral administration } \\
\mathbf{n}(\% \text { of prescriptions) }\end{array}$ \\
\hline Surgical infection & 74 & 5.5 (0.3 to 10.7$)$ & $137(15)$ & $123(89.8)$ \\
\hline Surgical prophylaxis & 92 & $6.8(1.7$ to 11.9$)$ & $123(17)$ & $95(77.2)$ \\
\hline Medical prophylaxis & 233 & $17.3(12.4$ to 22.16$)$ & $285(29)$ & $25(8.8)$ \\
\hline $\begin{array}{l}\text { Sepsis/CRBSI/CNS/febrile } \\
\text { neutropaenia }\end{array}$ & 246 & $18.2(13.4$ to 23.0$)$ & $385(22)$ & $371(96.4)$ \\
\hline URTI & 73 & $5.4(0.2$ to 10.6$)$ & $90(14)$ & $42(46.7)$ \\
\hline $\begin{array}{l}\text { LRTI/UTI/SSTI/joint bone/ } \\
\text { fever/GITI }\end{array}$ & 569 & 42.2 (38.1 to 46.3$)$ & $764(35)$ & $458(60.0)$ \\
\hline Other/unknown & 61 & 4.6 (0.0 to 9.7$)$ & $74(22)$ & $38(51.4)$ \\
\hline $\begin{array}{l}\text { Community-acquired } \\
\text { infection }\end{array}$ & 797 & 59.1 (55.7 to 62.5$)$ & $1121(34)$ & $774(69.1)$ \\
\hline Hospital-acquired infection & 211 & 15.7 (10.8 to 20.6$)$ & $298(28)$ & $240(80.5)$ \\
\hline $\begin{array}{l}\text { Other (prophylaxis or } \\
\text { unknown) }\end{array}$ & 340 & 25.2 (20.6 to 29.8$)$ & $439(34)$ & $138(31.4)$ \\
\hline Total & 1348 & & $1858(41)$ & \\
\hline
\end{tabular}




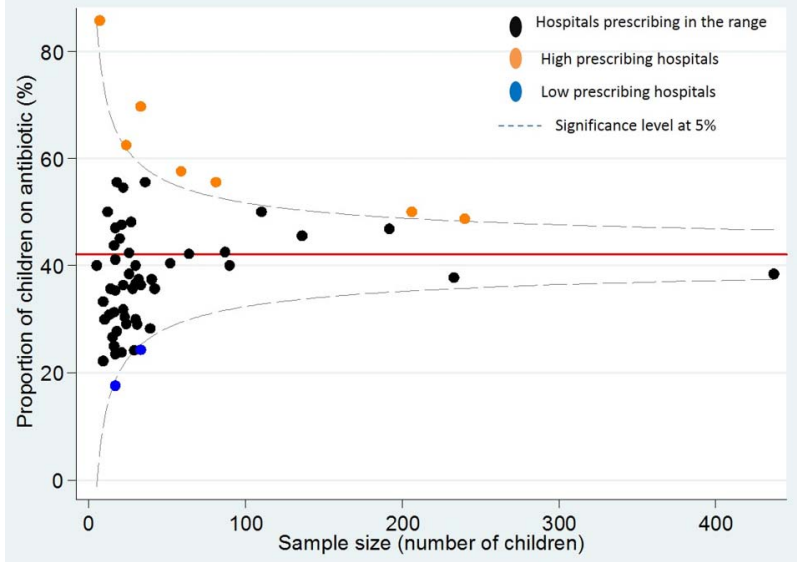

Figure 1 Funnel plot comparing hospital prescribing in the UK using proportion of children on antibiotics.

healthy children $(70.1 \%$ of the prescriptions), for surgical infections $(89.8 \%$ of the prescriptions) and for sepsis, CNS infections and febrile neutropaenia (96.4\% of the prescriptions) (table 1).

\section{Total usage of antibiotics in children in DDD/100 inpatients}

Table 2 illustrates the total usage of antibiotics in DDD/ 100 inpatients for each age category per type of hospital and specialty. The total amount of antibiotics used is slightly higher in tertiary hospitals than in district general hospitals (37.8 vs $30.7 \mathrm{DDD} / 100$ inpatients), except for children aged 1-6 years. The use of antibiotics is about twice as common in haematology-oncology-transplant units compared with other specialties, especially for patients aged above 12 years. For patients aged below 1 year, the use of antibiotics is substantially higher in PICU compared with other specialties.

The total prescribed antibiotics in DDD/100 inpatients per age band is shown in figure 2. A wide range of antibiotic use is observed among the 61 centres for patients aged between 12 and 18 years, whereas the three other groups show greater homogeneity between institutions in antibiotic usage. The total prescribed antibiotics are harmonised between district general hospitals and tertiary referral hospitals across the four age groups.

\section{Carbapenems and piperacillin-tazobactam}

Table 3 shows that among children receiving at least one antibiotic, the proportion of children on carbapenems was significantly higher in tertiary hospitals than in district general hospitals (respectively, $\mathrm{n}=54,6.0 \%$ vs $\mathrm{n}=7$, $2.0 \%, p=0.003)$. The same results were observed for the total amount of DDD/100 inpatients. Less than half of the children on carbapenems had at least one underlying disease recorded for district general hospitals, while more than 9 out of 10 had an underlying disease for tertiary hospitals. In district general hospitals, the general paediatric wards were the main prescribers of carbapenems as an empirical treatment, whereas in tertiary hospitals about $43 \%$ of the prescriptions were targeted and PICU were the main prescribers.

The amount of piperacillin-tazobactam in DDD/100 inpatients was also surprisingly twofold higher in district general hospitals than in tertiary hospitals. However, the proportion of children on piperacillin-tazobactam among all the children on antibiotics was much higher in tertiary hospitals. In district general hospitals, most of the patients were prescribed piperacillin-tazobactam in paediatric general wards as an empirical treatment when they had at least one underlying disease, whereas in tertiary hospitals piperacillin-tazobactam was prescribed in haematology-oncology-transplant wards in the presence of an underlying disease.

\section{DISCUSSION}

We describe a unique inpatient antibiotic prescribing data set from 61 paediatric units across the UK. Our results identified areas of potential improvement for appropriate prescribing at the patient level adjusting for risk factors (age, underlying diseases, infections, specialties), using the paediatric point prevalence method developed by the ARPEC project. Our results provide the baseline for future benchmarking to monitor national strategies for optimal antimicrobial prescribing in children, particularly the CQUIN NHS England scheme 2015/2016 for AMR.

A total of 1247 out of 3047 surveyed admitted children were on antibiotics in this study. The proportion of children receiving antibiotics showed a wide variation

Table 2 Total usage of antibiotics in DDD/100 inpatients in paediatric acute care settings across the UK, year 2011-2012

\begin{tabular}{|c|c|c|c|c|}
\hline & \multicolumn{4}{|c|}{ DDD/100 inpatients } \\
\hline & Aged $<1$ year & Aged $1-6$ years & Aged $7-11$ years & Aged $>12$ years \\
\hline District general hospitals, $n=958$ & 3.2 & 12.3 & 6.0 & 9.2 \\
\hline Tertiary referral hospitals, $n=2089$ & 4.0 & 10.5 & 7.1 & 16.2 \\
\hline General paediatric, $n=1477$ & 3.9 & 11.9 & 4.7 & 12.8 \\
\hline PICU, $n=226$ & 7.5 & 12.7 & 6.4 & 10.9 \\
\hline Paediatric surgery, $n=597$ & 2.1 & 9.0 & 10.7 & 15.7 \\
\hline Haematology-oncology-transplant, $n=144$ & 0.45 & 14.2 & 14.0 & 31.7 \\
\hline Others, $n=603$ & 4.3 & 9.7 & 6.3 & 11.9 \\
\hline Total & 32.9 & 64.8 & 118.3 & 207.5 \\
\hline
\end{tabular}




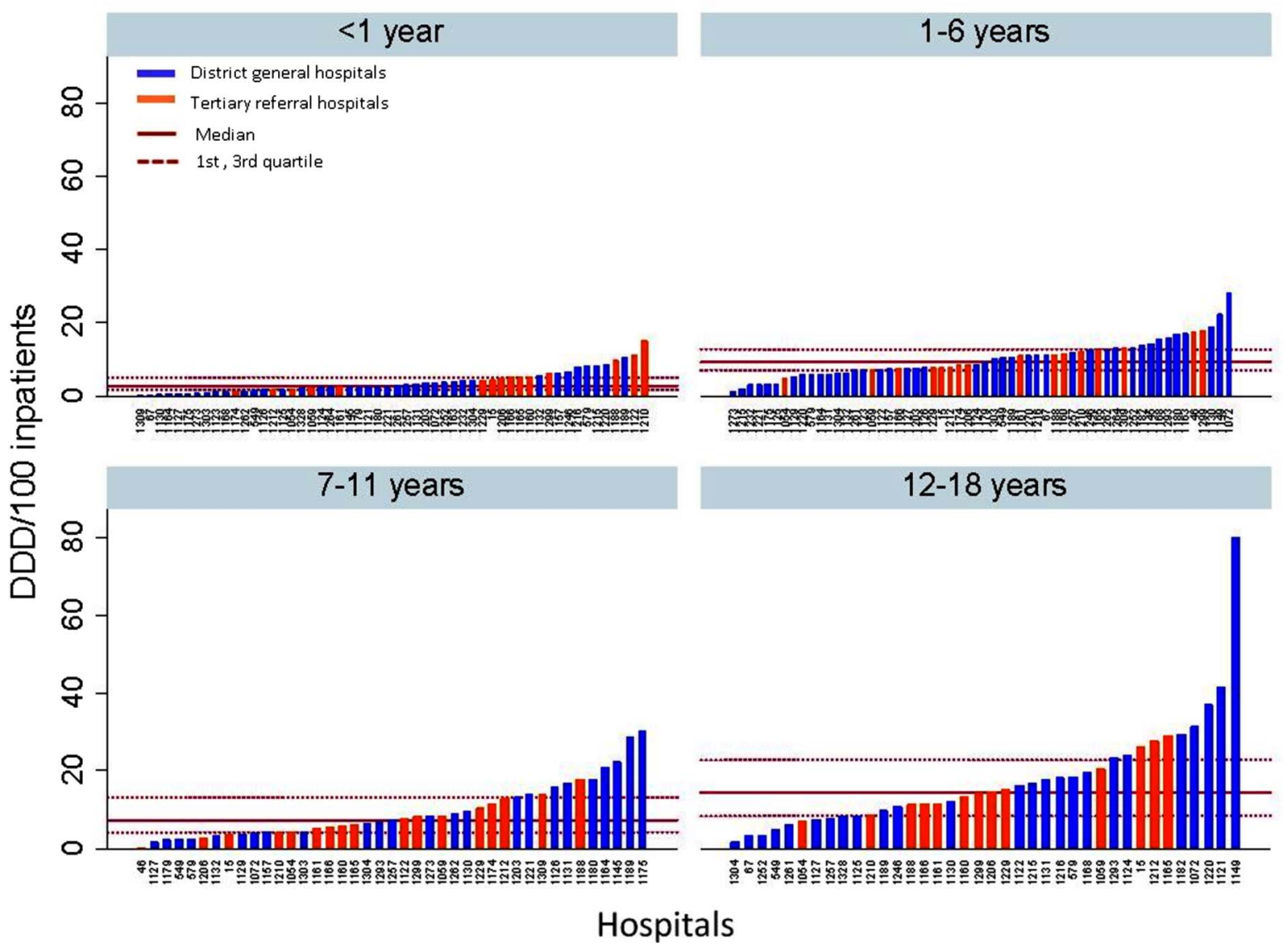

Figure 2 Total prescribed antibiotics (DDD/100 inpatients) per age class and type of hospital across the UK during the point prevalence survey in 2011-1012. DDD, defined daily doses.

Table 3 Total usage of carbapenems and piperacillin-tazobactam in paediatric acute care settings across the UK, year 2011-2012

\begin{tabular}{|c|c|c|c|c|}
\hline & \multicolumn{2}{|l|}{ Carbapenems } & \multicolumn{2}{|c|}{ Piperacillin-tazobactam } \\
\hline & $\begin{array}{l}\text { District general } \\
\text { hospitals ( } 349 \\
\text { children on } \\
\text { antibiotics) }\end{array}$ & $\begin{array}{l}\text { Tertiary referral } \\
\text { hospitals ( } 898 \\
\text { children on } \\
\text { antibiotics) }\end{array}$ & $\begin{array}{l}\text { District general } \\
\text { hospitals ( } 349 \\
\text { children on } \\
\text { antibiotics) }\end{array}$ & $\begin{array}{l}\text { Tertiary referral } \\
\text { hospitals ( } 898 \\
\text { children on } \\
\text { antibiotics) }\end{array}$ \\
\hline Total DDD/100 inpatients & 36.4 & 56.0 & 39.7 & 20.0 \\
\hline Total children, $\mathrm{n}(\%)^{\star}$ & $7(2.0)$ & $54(6.0)$ & $14(4)$ & $68(7.6)$ \\
\hline General paediatric, $n$ children $(\%) \dagger$ & $6(85.7)$ & $14(25.9)$ & $11(78.6)$ & $9(13.2)$ \\
\hline PICU, n children (\%) & $1(14.3)$ & $17(31.5)$ & 0 & $12(17.6)$ \\
\hline Paediatric surgery, n children (\%) & 0 & $6(11.1)$ & $3(21.4)$ & $7(10.3)$ \\
\hline $\begin{array}{l}\text { Haematology-oncology-transplant, } \\
\text { n children (\%) }\end{array}$ & 0 & $10(18.5)$ & 0 & $19(27.9)$ \\
\hline Others, $\mathrm{n}$ children (\%) & 0 & $7(13.0)$ & 0 & $21(30.9)$ \\
\hline $\begin{array}{l}\text { Underlying disease vs previously } \\
\text { healthy children, } n \text { children (\%)† }\end{array}$ & $3(42.9)$ & $49(90.7)$ & $12(85.7)$ & 67 (98.5) \\
\hline
\end{tabular}

between district general hospitals and tertiary referral hospitals, as well as a wide variation within both groups of hospitals. The presence of case mix and specialties, such as haematology-oncology-transplant and PICU, may be responsible for some of the differences observed in prescribing. Figure 1 highlighted that a total of $7 / 61$ $(11.5 \%)$ institutions, mainly the haematology-oncologytransplant and PICU units of the tertiary hospitals, were identified as potential 'high prescribers'. However, potential 'high prescribers' in general district hospitals 
were only general paediatric units with less than half of the patients having an underlying disease.

We also highlighted a proportion of patients on medical prophylaxis $(17.3 \%)$ similar to that in other countries $(16.9 \%$ in Italy and $14.8 \%$ on average worldwide) ${ }^{19}{ }^{26}$ Medical prophylaxis appeared to be one of the most common indications for antibiotic prescribing in children. The reason, duration and need for prophylaxis should be further assessed for quality improvement through ASPs across paediatric units in the UK, as it is in adult settings. ${ }^{27}$

The total usage of antibiotics in DDD/100 inpatients per age group showed a higher consumption in haematology-oncology-transplant units compared with the other specialties, except for those aged under 1 year receiving antibiotics in PICU. Children admitted to haematologyoncology-transplant units or to PICU were more likely to receive a combination of antibiotics than general and surgical paediatric patients, which may directly impact on exposure measured in DDD/1000 inpatients.

Carbapenems and piperacillin-tazobactam were mainly prescribed empirically, and to children with underlying conditions in tertiary hospitals. These results are expected and will serve as a benchmark in future evaluations. However, we did not predict that general paediatric units were high prescribers for these two drugs in district general and tertiary hospitals. With the spread of extended-spectrum $\beta$-lactamase producing Enterobacteriaceae in adults ${ }^{28}$ as well as in paediatrics over the past decade, ${ }^{29}$ and the increase of small outbreaks of multidrug-resistant organisms in UK paediatric hospitals, ${ }^{30}$ the prescribing pattern for these critical drugs may change in the future and needs to be better monitored, especially in the general paediatric units for previously healthy children.

There remains a lack of consensus regarding the optimal metric to assess paediatric antimicrobial use, which is an important limitation. The use of DDD/100 inpatients (DDD being defined as the amount of antibiotic prescribed for a $70 \mathrm{~kg}$ average adult weight for its main indication) proposed by CQUIN AMR is not a perfect measure, especially in children with a wide range of weights (from $5 \mathrm{~kg}$ in a 3 months old to over $100 \mathrm{~kg}$ in obese adolescents). Since DDD is weightdependent and dose-dependent, ${ }^{31}$ we decided to compare overall drug exposure using DDD/100 inpatients in age bands as proposed by Porta et al. ${ }^{24}$ Despite DDD/100 inpatients being advocated by the WHO Collaborating Centre for Drug Statistics and Methodology, 'days of therapy' could have advantages over DDD measures, because the impact of variation in absolute dose is limited for this metric. ${ }^{11}{ }^{31}$ However, longitudinal studies or access to electronic-prescribing systems for each hospital in the UK would be required to calculate this, which may not be realistic in the near future. ${ }^{32}$ For now, DDD/100 inpatients could be used to monitor changes within units over time as long as the case mix remains the same. While we have strongly promoted this study to include a large number of paediatric centres from a wide variety of different hospitals, wards and patient characteristics across the UK, only volunteer centres were recruited, with the potential for selection biases. Finally, the PPS methodology provided limited evidence on the temporal relationship between antimicrobial prescribing in children and covariates of interest.

In conclusion, we provide a robust baseline for antibiotic prescribing in hospitalised children in relation to current national stewardship efforts in the UK. Repeated PPS $^{33}$ needs to be part of the paediatric antibiotic stewardship strategy in order to identify prescribing trends over time, to evaluate the efficacy of ASPs and to tackle the issue of suboptimal antibiotic use, especially on antibiotic dosing. ${ }^{34}$ International standardised PPS with further linkage between antibiotic prescribing and resistance will be critical to characterise appropriate use of antibiotics in hospitalised children globally and to propose guidance on the management of paediatric infections taking into account resistance profiles.

\section{Author affiliations}

${ }^{1}$ NIHR Health Protection Research Unit Antimicrobial Resistance and Healthcare Associated Infection-Department of Primary Care and Public Health, Imperial College London, London, UK

${ }^{2}$ Paediatric Infection Diseases, St George's Hospital NHS Trust, London, UK

${ }^{3}$ Institute for Infection and Immunity-Paediatric Infectious Diseases Research Group, St. George's University of London, London, UK

${ }^{4}$ Paediatric Infectious Diseases and Immunology, Alder Hey Children's NHS Foundation Trust, Liverpool, UK

${ }^{5}$ Department of General Paediatrics, Evelina London Children's Hospital, London, UK

${ }^{6}$ Department of Infectious Diseases, St Mary's Hospital Imperial College Healthcare NHS Trust, London, UK

${ }^{7}$ Paediatric Infectious Diseases and Immunology, Southampton Children's Hospital, Southampton, UK

${ }^{8}$ Paediatric Infectious Disease and Immunology, University Hospitals Bristol NHS Foundation Trust, Bristol Royal Hospital for Children, Bristol, UK ${ }^{9}$ Department of Medical Microbiology, Vaccine \& Infectious Disease Institute (VAXINFECTIO) University of Antwerp, Antwerp, Belgium

${ }^{10}$ National Public Health Service for Wales, Cardiff, UK

Acknowledgements The authors thank all the members of the ARPEC Steering Committee; the management team based at St George's University of London, especially Helen Bird and Dr Tatiana Munera for their technical support; the ARPEC-PPS coordination team based at the Laboratory of Medical Microbiology, University of Antwerp, Antwerp, Belgium, especially Nico Drapier for the web application development. The authors also thank Jeff Aston, Dr Mitul Patel and Dr Alison Bedford Russell at the Birmingham Children's Hospital NHS Foundation Trust, Birmingham, UK; Dr Prakash Satodia at the University Hospitals Coventry and Warwickshire NHS Trust, Coventry, UK; Dr Mehdi Garbash at the County Durham \& Darlington NHS Foundation Trust (incorporating University Hospital North Durham and Darlington Memorial Hospital), Durham and Darlington, UK; Dr Alison Johnson at the Wye Valley NHS Trust, Hereford, UK; David Sharpe at the Alder Hey Children's NHS Foundation Trust, Liverpool, UK; Dr Christopher Barton at the Institute of Child Health, University of Liverpool, Liverpool, UK; Sara Arenas-Lopez, at the Evelina London Children's Hospital, London, UK; Dr Suzanne Luck at the Kingston Hospital NHS Foundation Trust, London, UK; Dr Paddy McMaster at the North Manchester General Hospital, Manchester, UK; Neil A Caldwell at the Wirral University Teaching Hospital NHS Foundation Trust, Merseyside, UK; Dr Andrew Lunn at the Nottingham Children's Hospital, Nottingham, UK; Dr Simon B Drysdale at the Oxford University 
Hospitals NHS Trust, Oxford, UK; Dr Rachel Howe at the Peterborough City Hospital, Peterborough, UK; Dr Tim Scorrer at the Queen Alexandra Hospital, Portsmouth, UK; Dr Florian Gahleitner at the Queen Alexandra Hospital, Portsmouth, UK; Dr Richa Gupt at the Royal Preston Hospital, Lancashire Teaching Hospitals, Preston, UK; Clare Nash at the Sheffield Children's NHS Foundation Trust, Sheffield, UK; Dr John Alexander at the University Hospital of North Midlands, Stoke on Trent, UK; Dr Mala Raman, at the Torbay Hospital, South Devon Health Care NHS Foundation Trust Torquay, UK; Dr Emily Bell at the Royal Cornwall Hospitals NHS Trust, Truro, Cornwall, UK; and Veena Rajagopal at the St George's NHS trust, London, UK and Fortis Escorts Heart Institute, Delhi, India.

Contributors MG, KD, JAB, AV, HG and MS had substantial contributions to the conception and the design of the work; MG, KD, SV, JAB, SP, EM, AR, $H L, S V P, J B, A V, M H, H G$ and MS participated in the acquisition and interpretation of data for the work. The data management was carried out by $A V$ and $M G$ and the data analysis by MG. MG, KD, SV and MS drafted the work and MG, KD, SV, JAB, SP, EM, AR, HL, SVP, JB, AV, MH, HG and MS revised it critically for important intellectual content; all authors gave the final approval of the version to be published and agree to be accountable for all aspects of the work in ensuring that questions related to the accuracy or integrity of any part of the work are appropriately investigated and resolved.

Funding The ARPEC project was co-funded by the European Commission Directorate General for Health and Consumers (DG SANCO) through the Executive Agency for Health and Consumers (agreement number-20091101; http://ec.europa.eu/eahc/). The October to November 2012 ARPEC-PPS was co-funded by PENTA (http://www.penta-id.org/). MG is funded by the National Institute for Health Research Health Protection Research Unit (NIHR HPRU) in Healthcare Associated Infection/Antimicrobial Resistance and the NIHR Imperial Biomedical Research Centre (BRC) at Imperial College London in partnership with Public Health England (PHE).

Disclaimer The views expressed are those of the author(s) and are not necessarily those of the National Health Service (NHS), the NIHR, the Department of Health or PHE.

\section{Competing interests None declared.}

Ethics approval UK Research Ethics Committee.

Provenance and peer review Not commissioned; externally peer reviewed.

Data sharing statement The additional unpublished data on antimicrobial prescribing for neonates and children are currently being published within the ARPEC project.

Open Access This is an Open Access article distributed in accordance with the terms of the Creative Commons Attribution (CC BY 4.0) license, which permits others to distribute, remix, adapt and build upon this work, for commercial use, provided the original work is properly cited. See: http:// creativecommons.org/licenses/by/4.0/

\section{REFERENCES}

1. Baquero F, Negri MC, Morosini MI, et al. Antibiotic-selective environments. Clin Infect Dis 1998;27(Suppl 1):S5-11.

2. Jacobson $\mathrm{KL}$, Cohen $\mathrm{SH}$, Inciardi JF, et al. The relationship between antecedent antibiotic use and resistance to extended-spectrum cephalosporins in group I beta-lactamase-producing organisms. Clin Infect Dis 1995;21:1107-13.

3. Davies SC, Gibbens N. UK five year antimicrobial resistance strategy 2013 to 2018. London: Department of Health, 2013

4. WHO. The evolving threat of antimicrobial resistance: options for action. Geneva: World Health Organisation, 2012.

5. CDC. Antibiotic resistance threats in the United States. Atlanta: Centres for Disease Control and Prevention, 2013.

6. Charani E, Holmes $\mathrm{AH}$. Antimicrobial stewardship programmes: the need for wider engagement. BMJ Qual Saf 2013;22:885-7.

7. Ashiru-Oredope D, Sharland M, Charani E, et al. Improving the quality of antibiotic prescribing in the NHS by developing a new antimicrobial stewardship programme: start smart-then focus. $J$ Antimicrob Chemother 2012;67(Suppl 1):i51-63.

8. Hersh AL, Beekmann SE, Polgreen PM, et al. Antimicrobial stewardship programs in pediatrics. Infect Control Hosp Epidemiol 2009;30:1211-17
9. Di Pentima MC, Chan S, Hossain J. Benefits of a pediatric antimicrobial stewardship program at a children's hospital. Pediatrics 2011;128:1062-70.

10. Gerber JS, Newland JG, Coffin SE, et al. Variability in antibiotic use at children's hospitals. Pediatrics 2010;126:1067-73.

11. Fridkin SK, Srinivasan A. Implementing a strategy for monitoring inpatient antimicrobial use among hospitals in the United States. Clin Infect Dis 2014;58:401-6.

12. Fortin E, Fontela PS, Manges AR, et al. Measuring antimicrobial use in hospitalized patients: a systematic review of available measures applicable to paediatrics. J Antimicrob Chemother 2014;69:1447-56.

13. Ibrahim OM, Polk RE. Benchmarking antimicrobial drug use in hospitals. Expert Rev Anti Infect Ther 2012;10:445-57.

14. Polk RE, Fox C, Mahoney A, et al. Measurement of adult antibacteria drug use in 130 US hospitals: comparison of defined daily dose and days of therapy. Clin Infect Dis 2007;44:664-70.

15. ARHAI. Advisory Committee on Antimicrobial Resistance and Healthcare Associated Infection (ARHAI) 4th Annual Report, February 2012-March 2013. Departement of Health, 2013:25.

16. ARHAl. Advisory Committee on Antimicrobial Resistance and Healthcare Associated Infection-Antimicrobial stewardship: "START SMART-THEN FOCUS” Guidance. London, UK: Department of Health, 2011:27.

17. NHS. Commissioning for Quality and Innovation (CQUIN)Guidance for 2016/17. 2016. Available from https://www.england. nhs.uk/wp-content/uploads/2016/03/cquin-guidance-16-17-v3.pdf.

18. Amadeo B, Zarb P, Muller A, et al. European Surveillance of Antibiotic Consumption (ESAC) point prevalence survey 2008: paediatric antimicrobial prescribing in 32 hospitals of 21 European countries. J Antimicrob Chemother 2010;65:2247-52.

19. Versporten A, Bielicki J, Drapier N, et al. The Worldwide Antibiotic Resistance and Prescribing in European Children (ARPEC) point prevalence survey: developing hospital-quality indicators of antibiotic prescribing for children. J Antimicrob Chemother 2016;71:1106-17.

20. Henderson KL, Muller-Pebody B, Johnson AP, et al. First set-up meeting for Antibiotic Resistance and Prescribing in European Children (ARPEC). Euro Surveill 2009;14:pii: 19404 .

21. Versporten $A$, Sharland $M$, Bielicki J, et al. The antibiotic resistance and prescribing in European Children project: a neonatal and pediatric antimicrobial web-based point prevalence survey in 73 hospitals worldwide. Pediatr Infect Dis J 2013;32:e242-53.

22. WHO. Guidelines for ATC classification and DDD assignment 2013. Oslo: WHO Collaborating Centre for Drug Statistics Methodology, 2012

23. Porta A, Esposito S, Menson E, et al. Off-label antibiotic use in children in three European countries. Eur J Clin Pharmacol 2010;66:919-27.

24. Porta A, Hsia Y, Doerholt K, et al. Comparing neonatal and paediatric antibiotic prescribing between hospitals: a new algorithm to help international benchmarking. J Antimicrob Chemother 2012;67:1278-86.

25. Spiegelhalter DJ. Funnel plots for comparing institutional performance. Stat Med 2005;24:1185-202.

26. De Luca M, Dona $D$, Montagnani $C$, et al. Antibiotic prescriptions and prophylaxis in Italian children. is it time to change? Data from the ARPEC Project. PLOS ONE 2016;11:e0154662.

27. Bruce J, MacKenzie FM, Cookson B, et al. Antibiotic stewardship and consumption: findings from a pan-European hospital study. $J$ Antimicrob Chemother 2009;64:853-60.

28. ECDC. European Centre for Disease Prevention and Control (ECDC)- EARS-net database. Secondary European Centre for Disease Prevention and Control (ECDC)- EARS-net database. 2015 http://www.ecdc.europa.eu/en/healthtopics/antimicrobial_resistance/ database/Pages/database.aspx

29. Lukac PJ, Bonomo RA, Logan LK. Extended-spectrum beta-lactamase-producing Enterobacteriaceae in children: old foe, emerging threat. Clin Infect Dis 2015;60:1389-97.

30. Drew RJ, Turton JF, Hill RL, et al. Emergence of carbapenem-resistant Enterobacteriaceae in a UK paediatric hospital. J Hosp Infect 2013;84:300-4.

31. Gravatt LA, Pakyz AL. Challenges in measuring antibiotic consumption. Curr Infect Dis Rep 2013;15:559-63.

32. Ahmed Z, McLeod MC, Barber N, et al. The use and functionality of electronic prescribing systems in English acute NHS trusts: a cross-sectional survey. PLOS ONE 2013;8:e80378.

33. Willemsen I, Groenhuijzen A, Bogaers D, et al. Appropriateness of antimicrobial therapy measured by repeated prevalence surveys. Antimicrob Agents Chemother 2007:51:864-7.

34. Lestner JM, Versporten A, Doerholt K, et al. Systemic antifungal prescribing in neonates and children: outcomes from the Antibiotic Resistance and Prescribing in European Children (ARPEC) Study. Antimicrob Agents Chemother 2015;59:782-9. 